INTERSTITIAL LUNG DISEASE

\title{
Lymphangioleiomyomatosis: a case-control study of perinatal and early life events
}

\author{
C I Whale, S R Johnson, K G Phillips, S A Newton, S A Lewis, A E Tattersfield
}

Thorax 2003;58:979-982

See end of article for authors' affiliations

.....................

Correspondence to: Professor A E Tattersfield Clinical Sciences Building, City Hospital, Hucknall

Road, Nottingham NG5

IPB, UK:

anne.tattersfield@

nottingham.ac.uk
Background: Lymphangioleiomyomatosis (LAM), a rare disease affecting women, is caused by somatic mutations in the tuberous sclerosis complex genes.

Methods: A case-control questionnaire study was carried out examining parental and family history, prenatal events, and early life events to try to shed light on the aetiology of the condition. Forty five patients identified from a national LAM register completed a questionnaire and 31 were compared with 117 age and sex matched control subjects using conditional logistic regression.

Results: No differences were found in perinatal events, childhood infections, and parental or family history, except that patients were more likely to be an only child (odds ratio (OR) 4.3 (95\% confidence interval (CI), 1.5 to 11.8)) and have a relative with uterine fibroids (OR 4.2 (1.4 to 13)). Patients with LAM had had fewer pregnancies and fewer children but no differences in miscarriage rates. A non-matched analysis using all 45 cases and 117 controls gave similar results.

Conclusions: No features in the family history, perinatal events, or early life events were detected that were associated with having LAM. Being more likely to be an only child and having an increased family history of uterine fibroids may, if confirmed, indicate some differences in reproductive function within the families of affected individuals. ymphangioleiomyomatosis (LAM), a disease of unknown aetiology, affects only women. Abnormal smooth muscle -cells cause lung cysts, airways obstruction, pneumothoraces, and chylous pleural effusions; renal angiomyolipomas and lymphangioleiomyomas may also be present. ${ }^{1-3}$ Hormonal factors could be involved, as LAM has a tendency to deteriorate during pregnancy ${ }^{4}$ and in women taking hormone replacement therapy or exogenous oestrogens, ${ }^{5}$ and the rate of decline appears to slow after the menopause and with progesterone treatment. ${ }^{6}$

LAM occurs sporadically and also in patients with tuberous sclerosis, ${ }^{8}$ which can be inherited as an autosomal dominant disorder. The two genes responsible for tuberous sclerosistuberous sclerosis complex (TSC) 1 and TSC2-are abnormal in LAM tissue, whether LAM is associated with tuberous sclerosis or not. ${ }^{10}$ Isolated LAM may therefore arise from somatic mutations. As gene mutations may be related to prenatal, perinatal, or early life events, we sought to determine whether such events differed between LAM patients and control subjects.

We carried out a national case-control study comparing family history, perinatal events, and early life events between patients with LAM and controls. We also asked about patients' height, as a marker of intrauterine growth and early development, and about their personal smoking and reproductive history, as a follow up to our 1990 case-control study which looked at the use of the oral contraceptive pill. ${ }^{11}$

\section{METHODS}

Patients with LAM were identified from the 60 patients on the Nottingham LAM register, and we contacted the 51 who had agreed to help with research. The register was started in 1995 by writing to all respiratory physicians in the United Kingdom to ask for names of patients with lymphangioleiomyomatosis. Further patients have been added through personal contacts or LAM patient groups. After receiving a completed questionnaire, we asked the patient's general practitioner to identify six women in the practice who were closest in age to the patient to be control subjects, and to send them the questionnaire with a letter explaining the study rationale and need for control subjects. Nottingham City Hospital ethics committee approved the study.

\section{Questionnaire}

Patients and control subjects completed identical questionnaires except that when the case questionnaire asked about the effects of LAM or events after the onset of LAM the control question asked about any medical condition or events over the last 10 years (tables 1 and 2). The questionnaire asked about illnesses in the family, including those that might be related to tuberous sclerosis, the mother's reproductive history, perinatal and early life events, childhood infections, therapeutic drug use, and obstetric or gynaecological problems. A second letter was sent to patients and general practitioners who failed to reply.

\section{Data analysis}

Replies were anonymised and coded independently. Data were entered into SPSS for cross tabulation of case-control status against each risk factor. We undertook a matched analysis on all patients for whom there was at least one control subject, using conditional logistic regression in EGRET for Windows (Cytel Software Corporation) to give odds ratios (OR) and 95\% confidence intervals (CI). We compared this analysis with an unmatched comparison of all cases and controls to assess whether the exclusion of cases for whom we were unable to obtain a control affected our results. A probability ( $p$ ) value of $<0.05$ was considered statistically significant.

\section{RESULTS}

Of the 51 patients with LAM who were sent the questionnaire, 45 (88\%) completed it (mean (SD) age 48.7 (9.4) years), including 11 from our previous case-control study. ${ }^{11}$ Thirty eight of the 45 general practices (84\%) agreed to send questionnaires to control subjects, and 117 of 228 potential 
Table 1 Family history, parental details, perinatal events, and early life events: responses in cases and controls

\begin{tabular}{|c|c|c|c|c|}
\hline & Cases $(n=31)$ & Controls $(n=117)$ & OR $(95 \% \mathrm{Cl})$ & p Value \\
\hline \multicolumn{5}{|l|}{ Family events } \\
\hline Is your mother alive? & $21 / 31$ & $69 / 116$ & $1.6(0.6$ to 4.3$)$ & 0.35 \\
\hline Is your father alive? & $15 / 30$ & $45 / 112$ & $1.5(0.6$ to 3.8$)$ & 0.4 \\
\hline Did your father smoke? & $22 / 30$ & $89 / 110$ & $0.7(0.3$ to 1.8$)$ & 0.44 \\
\hline Did your mother smoke? & $14 / 30$ & $64 / 113$ & $0.7(0.3$ to 1.7$)$ & 0.48 \\
\hline Mother take medicines during pregnancy? & $5 / 24$ & $8 / 76$ & $2.8(0.8$ to 10.0$)$ & 0.11 \\
\hline Mother had miscarriages? & $6 / 29$ & $30 / 98$ & $0.7(0.2$ to 1.8$)$ & 0.4 \\
\hline Maternal pregnancy problems? & $5 / 27$ & $22 / 96$ & $0.9(0.3$ to 2.6$)$ & 0.79 \\
\hline Family history kidney problems? & $5 / 30$ & $17 / 114$ & $1.0(0.3$ to 3.1$)$ & 0.94 \\
\hline Family history fits/epilepsy? & $6 / 30$ & $20 / 114$ & $1.2(0.4$ to 3.4$)$ & 0.74 \\
\hline Family history learning difficulties? & $4 / 30$ & $10 / 114$ & $1.4(0.4$ to 4.9$)$ & 0.64 \\
\hline Has anyone in the family got fibroids? & $10 / 29$ & $14 / 109$ & $4.2(1.4$ to 12.9$)$ & 0.01 \\
\hline $\begin{array}{l}\text { Are you an only child? } \\
\text { Early life events }\end{array}$ & $9 / 31$ & $11 / 116$ & $4.3(1.5$ to 11.8$)$ & 0.005 \\
\hline $\begin{array}{l}\text { Did you take medicines as a child? } \\
\text { ( }<12 \text { years) }\end{array}$ & $8 / 31$ & $7 / 115$ & $5.9(1.7$ to 19.9$)$ & 0.004 \\
\hline Were you a healthy child? & $25 / 31$ & $112 / 117$ & $0.3(0.1$ to 0.9$)$ & 0.03 \\
\hline Did you have measles? & $26 / 30$ & $82 / 99$ & $2.3(0.5$ to 11.4$)$ & 0.3 \\
\hline Did you have mumps? & $17 / 29$ & $54 / 115$ & $1.2(0.5$ to 11.4$)$ & 0.72 \\
\hline Did you have whooping cough? & $7 / 28$ & $28 / 101$ & $1.1(0.4$ to 3.6$)$ & 0.84 \\
\hline Did you have chicken pox? & $28 / 30$ & $98 / 108$ & $0.9(0.2$ to 4.3$)$ & 0.84 \\
\hline Did you have rubella? & $16 / 27$ & $61 / 88$ & $0.8(0.3$ to 2.1$)$ & 0.63 \\
\hline Other childhood illnesses? & $15 / 31$ & $45 / 114$ & $1.5(0.7$ to 3.3$)$ & 0.31 \\
\hline Any drugs in adolescence? & $4 / 30$ & $9 / 115$ & $1.6(0.4$ to 5.9$)$ & 0.48 \\
\hline Any childhood operations? & $12 / 31$ & $28 / 115$ & $2.4(0.9$ to 6.2$)$ & 0.09 \\
\hline
\end{tabular}

control subjects (51\%) responded (mean age 49.3 (8.4) years). Thirty one patients had at least one matched control subject. Details of the 31 patients and their control subjects with respect to family history, perinatal events, and early life events are given in table 1 .

Only the results of the matched analysis are presented, as the odds ratios and significance levels for the unmatched analysis of all cases and controls were very similar.

\section{Parents and families}

One LAM patient and one control had been adopted. There was no difference between the two groups with respect to parental social class distribution $(p=0.2)$, the number of parents who had smoked or total pack years of smoking, or the numbers of either parent who had died and the causes of death. Parental occupations varied widely for both groups, with no clustering of any particular type of occupation among the parents of patients with LAM.

The incidence of kidney problems, fits, epilepsy, or learning difficulties within the family was similar for the two groups; more LAM patients reported a family member with uterine fibroids (OR 4.2 (95\% CI, 1.4 to 12.9)).

\section{Perinatal events, childhood, and adolescence}

None of the mothers of subjects in either group had used fertility treatment. The mothers did not differ in problems or therapeutic drug use during pregnancy, miscarriage rates, premature or late births, or in the weight of their babies. More patients with LAM were an only child (OR 4.3 ( 1.5 to 11.8)). Among those who were not an only child there was no difference in the number of siblings.

There was no difference in incidence of measles, mumps, pertussis, varicella, rubella, or other childhood illnesses between patients and control subjects. Fewer LAM respondents said that they were healthy children, however (OR 0.3 $(0.08$ to 0.86$))$, and more had taken regular medicines as a child $(<12$ years). This covered a range of drugs, and antimalarials-taken by two subjects-were the only class of drug to have been taken regularly by more than one patient. There was no difference in medicine use during adolescence. Menarche occurred at a similar age in both groups.

\section{Experiences as an adult}

These findings are given in table 2. Twenty patients with LAM were not menstruating; of these, 18 were taking

Table 2 Experiences as an adult: responses for cases and controls

\begin{tabular}{lllll}
\hline & Cases $(\mathbf{n = 3 1 )}$ & Controls ( $\mathbf{n = 1 1 7 )}$ & OR (95\% Cl) & p Value \\
\hline Have your periods stopped? & $20 / 31$ & $44 / 114$ & $6.1(1.9$ to 19.7$)$ & 0.003 \\
Are your periods regular? & $8 / 12$ & $60 / 73$ & $0.5(0.1$ to 2.2$)$ & 0.36 \\
Ever taken the pill? & $25 / 31$ & $88 / 116$ & $1.5(0.6$ to 4.2$)$ & 0.4 \\
Have you ever been pregnant? & $22 / 31$ & $105 / 118$ & $0.3(0.1$ to 0.7$)$ & 0.01 \\
Problems getting pregnant? & $8 / 21$ & $16 / 97$ & $3.2(1.0$ to 9.6$)$ & 0.04 \\
Ever had a miscarriage? & $10 / 26$ & $42 / 115$ & $1.2(0.5$ to 2.8$)$ & 0.7 \\
Any fertility treatment? & $4 / 28$ & $7 / 113$ & $2.9(0.6$ to 13.2$)$ & 0.17 \\
Pregnancy complications? & $11 / 22$ & $34 / 115$ & $2.3(0.9$ to 6.0$)$ & 0.08 \\
Has LAM/a medical condition affected & $8 / 24$ & $6 / 114$ & $5.7(1.8$ to 17.8$)$ & 0.003 \\
your decisions to have children? & & & & \\
Has LAM/a medical condition caused & $9 / 23$ & $7 / 110$ & $8.1(2.4$ to 26.9) & 0.001 \\
you to have fewer children? & $8 / 30$ & $63 / 116$ & $0.3(0.1$ to 0.8$)$ & 0.01 \\
Have you smoked? & & & & \\
\hline Cl, confidence interval; OR, odds ratio. & & & &
\end{tabular}


progesterone treatment. The pattern of menstruation was similar in those still menstruating. There was no difference in the proportion of subjects who had taken the oral contraceptive pill or the age it was started; patients with LAM had taken it for a shorter time $(6.2 \vee 9.1$ years $)$, although the difference was not significant.

Patients with LAM were less likely to have been pregnant and had had fewer pregnancies $(p=0.04)$ and fewer children than control subjects (OR per child $=0.5(0.3$ to 0.8$)$ ). There was no difference between groups for miscarriage or termination rates, problems conceiving, complications during pregnancy, or the use of fertility treatment. One third of patients said that LAM had caused them to have fewer children than they had wanted.

There was no difference in height between the two groups $(1.62 v 1.63 \mathrm{~m}$ ) but patients with LAM tended to be lighter than control subjects $(63.8 v 68.4 \mathrm{~kg}, \mathrm{p}=0.2)$. Fewer patients with LAM had ever smoked and they had smoked for a shorter time than the control group ( $13 v 20$ years); there was no difference in daily cigarette consumption or pack years among current smokers, however.

\section{DISCUSSION}

LAM occurs sporadically and in patients with tuberous sclerosis. ${ }^{812} 13$ Loss of heterozygosity for TSC1 and TSC2 genes has been seen in lung tissue and angiomyolipomas from patients with sporadic LAM, ${ }^{9}$ causing speculation that sporadic LAM results from gene mutations associated with perinatal or early life events, possibly modified by hormonal factors.

This is the first case-control study to explore prenatal and early life events in lymphangioleiomyomatosis. Studying the aetiology of rare diseases is difficult and limited by the number of patients available. A national register for UK patients with LAM was set up in 1995 and updated regularly through contacts with doctors and patient self help groups. We were able, therefore, to send a questionnaire to all 51 patients known to have LAM in 1999 in the UK who were willing to help with research. We received a reply from 45 patients, and obtained at least one control subject for two thirds of the respondents. This was a gratifying response considering the sensitive nature of the questions in the questionnaire. We sent questionnaires to six controls, in order to attain around four controls for each case and to maximise study power. ${ }^{14}$ We have presented the results of the matched analysis on 31 patients, although the unmatched analysis on all 45 patients and control subjects gave very similar results. The questionnaires appeared to have been completed carefully by both groups. Nevertheless recall bias would have influenced some responses as LAM respondents may have been more motivated and delved more deeply to identify events within the family or in their early life.

The questionnaire asked about events that might shed light on the aetiology of LAM. We found no evidence that parental smoking, occupation and cause of death, perinatal events, or common illnesses in early childhood were involved. The study did not have sufficient power to exclude some contribution from these factors but suggests they are unlikely to be making a major contribution to the aetiology. Some of the findings, however, merit discussion.

Although none of the features associated with tuberous sclerosis-such as fits-was more common among the families of patients with LAM, there was an increased incidence of uterine fibroids (leiomyomas). Uterine fibroids are not a well recognised feature of LAM, although a nonsignificant increase was seen among patients with LAM in our previous study. ${ }^{11}$ The increased incidence could be a result of recall bias, although uterine leiomyomas are oestrogen sensitive benign smooth muscle tumours and the possibility of shared origin has been suggested. ${ }^{15}$ An increase in uterine fibroids could indicate some difference in hormonal balance or growth factors among relatives of patients with LAM.

Patients with LAM were more likely to be an only child. This could reflect problems with conception among parents of patients with LAM, although there was no increase in problems during pregnancy or in miscarriage rates. The increase in singleton children should be confirmed as this and the increase in uterine fibroids were the only findings to suggest any prenatal influence on the development of LAM.

There was no difference in early childhood illnesses between the two groups, nor in adult height, suggesting normal intrauterine growth and early development in patients with LAM. Somewhat fewer LAM patients described themselves as being well during childhood, and they were more likely to take regular medicines. LAM was not, however, associated with any particular type of drug, so the difference may reflect chance or recall bias. We cannot exclude an effect from a drug that was used for short periods, however, as we only asked about regular medicines, assuming that recall of occasional treatments would be inaccurate. We found no difference in the number of patients who had used the oral contraceptive pill, as in previous studies. ${ }^{11} 16$

Several of the differences between LAM patients and control subjects were expected, including having fewer pregnancies and fewer children, which several attributed to decisions made after LAM was diagnosed. The shorter use of the contraceptive pill was expected as women with LAM are commonly advised to stop exogenous oestrogens.

\section{Conclusions}

This is the first case-control study to look at early life events in patients with LAM. The only differences between families and parents of patients with LAM and controls were an increase in uterine fibroids among family members and an increase in singleton children among LAM parents. Although more LAM patients took regular medicines, the numbers were small and no specific drugs or childhood illnesses were associated with LAM.

\section{ACKNOWLEDGEMENTS}

We thank the general practitioners, patients, and control subjects who helped with the study, Pat Stephens for anonymising the data, Jan Johnson for providing details from the LAM register, and Richard Hubbard for comments on the manuscript.

Supported by The LAM Trust (Now LAM Action)

\section{Authors' affiliations}

C I Whale, K G Phillips, S A Newton, S A Lewis, A E Tattersfield,

Division of Respiratory Medicine, City Hospital, Nottingham, UK

S R Johnson, Division of Therapeutics, University Hospital, Nottingham

\section{REFERENCES}

1 Corrin B, Liebow AA, Friedman PJ. Pulmonary lymphangioleiomyomatosis. Am J Pathol 1975;79:348-82.

2 Johnson S. Rare diseases. 1. Lymphangioleiomyomatosis: clinical features, management and basic mechanisms. Thorax 1999:54:254-64.

3 Chu SC, Horiba K, Usuki J, et al. Comprehensive evaluation of 35 patients with lymphangioleiomyomatosis. Chest 1999;115:1041-52.

4 Johnson SR, Tattersfield AE. Clinical experience of lymphangioleiomyomatosis in the UK. Thorax 2000;55:1052-7.

5 Yano S. Exacerbation of pulmonary lymphangioleiomyomatosis by exogenous oestrogen used for infertility treatment. Thorax 2002;57:1085-6.

6 Taylor JR, Ryu J, Colby TV, et al. Lymphangioleiomyomatosis. Clinical course in 32 patients. N Eng J Med 1990;323:1254-60.

7 Johnson SR, Tattersfield AE. Decline in lung function in lymphangioleiomyomatosis: relation to menopause and progesterone treatment. Am J Respir Crit Care Med 1999;160:628-33.

8 Castro M, Shepherd CW, Gomez MR, et al. Pulmonary tuberous sclerosis. Chest 1995; 107:189-95. 
9 Carsillo T, Astrinidis A, Henske EP. Mutations in the tuberous sclerosis complex gene TSC2 are a cause of sporadic pulmonary lymphangioleiomyomatosis. Proc Natl Acad Sci USA 2000;97:6085-90.

10 Sato T, Seyama K, Fujii H, et al. Mutation analysis of the TSC1 and TSC2 genes in Japanese patients with pulmonary lymphangioleiomyomatosis. J Hum Genet 2002;47:20-8.

11 Wahedna I, Cooper S, Williams J, et al. Relation of pulmonary lymphangioleiomyomatosis to use of the oral contraceptive pill and fertility in the UK: a national case control study. Thorax 1994;49:910-14.

12 Costello LC, Hartman TE, Ryu JH. High frequency of pulmonary lymphangioleiomyomatosis in women with tuberous sclerosis complex. Mayo Clin Proc 2000;75:591-4.
13 Franz DN, Brody A, Meyer C, et al. Mutational and radiographic analysis of pulmonary disease consistent with lymphangioleiomyomatosis and micronodular pneumocyte hyperplasia in women with tuberous sclerosis. Am J Respir Crit Care Med 2001;164:661-8.

14 Gail M, Williams R, Byar DP, et al. How many controls? J Chronic Dis 1976;29:723-31

15 McCormack F, Smolarek T, Menon AG. Lymphangioleiomyomatosis and leiomyoma. Possibility of shared genetic origins? In: Moss J, ed. LAM and other diseases categorised by smooth muscle proliferation. New York: Marcel Dekker, 1999:373-86.

16 Oberstein EM, Fleming LE, Gomez-Marin O, et al. Pulmonary lymphangioleiomyomatosis (LAM): examining oral contraceptive pills and the onset of disease. J Womens Health 2003;12:81-5.

\section{LUNG ALERT}

Lung deposition of colomycin depends on the nebuliser used

$\Delta$ Byrne NM, Keavey PM, Perry JD, et al. Comparison of lung deposition of colomycin using the HaloLite and the Pari LC Plus nebulisers in patients with cystic fibrosis. Arch Dis Child 2003;88:715-8

nhalation of antibiotics against Pseudomonas aeruginosa slows the decline in lung function and increases survival in patients with cystic fibrosis. The Pari LC Plus is the breath

enhanced nebuliser most commonly used for administering aerosolised antibiotics. Drug delivery can be lengthy and unpredictable. The HaloLite analyses the patient's respiratory cycle, delivering a pulse of drug during the initial phase of inspiration.

This randomised crossover trial examined the delivery of nebulised colomycin by the HaloLite and Pari LC Plus nebulisers in 15 patients with cystic fibrosis. Patients were initially assessed for lung deposition of 1 mega unit of radiolabelled colomycin following nebulisation to dryness with the Pari and following a l button press dose from the HaloLite (manufacturer's recommended dose). Sputum colomycin levels and Pseudomonas load were assayed after 1 week with each nebuliser in turn.

There was greater lung uptake of colomycin with the Pari nebuliser, but lung uptake calculated as a percentage of total drug used was significantly lower and nebulisation time was longer. Sputum levels of colomycin were higher following use of the Pari nebuliser but there was no significant difference in sputum Pseudomonas loads between the delivery systems, although the authors accept this may represent a less reliable method of measurement than the radionuclide assay.

The concept of adaptive aerosol delivery is advantageous, but dose modification may be required for the HaloLite to improve effectiveness. This study emphasises the need for objective assessment of individual nebuliser devices with specific drugs used in patients with cystic fibrosis.

D Simcock 